\title{
Hyperbolic two-temperature generalized thermoelasticity with fractional order strain of solid cylinder
}

\author{
Alaa A. El-Bary \\ Basic and Applied Science Institute, Arab Academy for Science, Technology and Maritime Transport, \\ P.O. Box 1029, Alexandria, Egypt \\ National Committee for Mathematics, Academy of Scientific Research and Technology, Cairo, Egypt \\ E-mail: aaelbary@aast.edu \\ Received 26 March 2021; received in revised form 6 July 2021; accepted 6 August 2021 \\ DOI https://doi.org/10.21595/jets.2021.21969 \\ Check for updates \\ Copyright $(C 2021$ Alaa A. El-Bary. This is an open access article distributed under the Creative Commons Attribution License, which \\ permits unrestricted use, distribution, and reproduction in any medium, provided the original work is properly cited.
}

\begin{abstract}
A novel mathematical model of thermoelastic of a homogenous isotropic solid cylindrical infinite medium has been constructed in this paper. Thermally shocked is the bounding surface of the cylinder. In the sense of the hyperbolic two-temperature generalized thermoelasticity with fractional stress theory, the governing equations have been taken. Different values of the fractional order and two-temperature parameters have shown numerical results for the dynamical and conductive temperature increment, strain, displacement, and average stress, which are graphically applicable to all the functions studied. The fractional-order parameter has significant effects on stress and displacement distributions, while it has little effect on the dynamical and conductive temperatures increment and significant effects on all studied functions as well as on the two-temperature parameter. The two-temperature hyperbolic model is precious and effective.
\end{abstract}

Keywords: hyperbolic two-temperature generalized thermoelasticity, cylindrical body, fractional-order strain, two-temperature generalized thermoelasticity.

\section{Nomenclature}

$C_{E}$

$c_{o}=\sqrt{\frac{\lambda+2 \mu}{\rho}}$

$e$

K

$T, T_{0}$

$t$

$u$

$\alpha_{T}$

$\alpha$

$\beta=\left(\frac{\lambda+2 \mu}{\mu}\right)^{1 / 2}$

$\gamma=(3 \lambda+2 \mu) \alpha_{T}$

$\varepsilon=\frac{\gamma}{\rho C_{E}}$

$\varepsilon_{1}=\frac{\gamma T_{o}}{\mu}$

$\eta=\frac{\rho C_{E}}{K}$

$\lambda, \mu$
Specific heat at constant strain

Longitudinal wave speed

The volumetric strain

Thermal conductivity

Temperature and reference temperature

Time

The displacement functions

Coefficient of linear thermal expansion

Fractional-order parameter 

$\rho$
Density
$\sigma_{i j}$
Components of the stress tensor
$\tau_{o}, \tau$
Thermal and mechanical relaxation times

\section{Introduction}

Finding the best mathematical model that simulates the behavior of these materials close to experience is one of the key problems in materials and solid sciences. The mathematical model produces results that are completely consistent with experimental findings, and researchers in this area are primarily interested in the physical behavior of these compounds. Various mathematical models have been proposed by scientists and researchers to explain the propagation of mechanical waves and heat in solid and elastic materials. However, not all these models are suitable, since one of the metrics of an ideal model is to include the speed of progression of mechanical and thermal waves with finite values as experimental results. Mathematical models of thermomechanical transformation through elastic materials involve a large amount of space that cannot be included in a single study. However, new models that seek to demonstrate new observations that are very close to laboratory studies and are generally consistent with the physical behavior of thermo-elastic materials should be discussed.

Chen and Gurtin [1] have introduced a thermoelastic theory based on two different temperatures, conductive and dynamic temperature. The difference between these two temperatures is proportional to the heat source. Warren and Chen [2] studied wave propagation in the two-temperature thermo-elasticity principle. However, there is no investigation of this theory until Youssef [3] has updated this theory and introduced a two-temperature generalized thermo-elasticity model. Youssef and Elsibai enhanced the two-temperature thermoelasticity principle without a Green-Naghdi model energy dissipation [4]. Youssef and many other authors have used this model in many applications and research [5-8]. Youssef and El-Bary approved that the two-temperature generalized thermoelasticity model does not provide a finite speed of propagation of the thermal waves [9]. Youssef and El-Bary have therefore updated the model and have introduced a new two-temperature model based on different heat piping rules, known as hyperbolic, generalized 2-temperature thermoelasticity [9].

Youssef suggested in this model that the difference between the conductive temperature acceleration and the acceleration of the thermal temperature during the material transition is proportionate to the heater supply. The speed of the spread of thermal waves is limited within this model.

Additional new models of thermoelasticity based on the fractional calculus principle were introduced. Magin and Royston [10] have introduced the first model that applies the fractional deformation derivative characterizing the material's behavior. The zero-order of the derivative is for a Hookean solid while the one-order is for a Newtonian fluid. The thermoelastic and thermoviscoelastic materials are sitting in the intermediate range with a fractional order parameter between zero and one [10]. C Li et al. studied the generalized thermoelastic diffusion problems with fractional-order strain $[11,12]$. Li, Chenlin, et al. studied the generalized piezoelectric thermoelasticity problems with strain rate and transient thermo-electromechanical responses analysis [13].

Recently, many applications used the fractional-order definition with various construction and different models [14-22]. Depending on the fractional strain, Youssef [23] presented another new theory of generalized thermoelectricity. It is seen as another shift in the stress-strain relationship between Duhamel and Neumann. Youssef solved an application of thermoelasticity with fractional order strain for a one-dimensional half-space, in the context of models of Biot, Lord-Shulman, Green-Lindsay, and Green-Naghdi type II [23]. Youssef solved many applications of thermoelasticity of infinite thermoelastic cylindrical medium $[8,24]$.

In the present investigation, we will study the induced conductive temperature, dynamic temperature, deformation, displacement, and stress fields in a thermoelastic one-dimensional 
infinite cylindrical body. The work under the purview of the theory of thermoelasticity with fraction order strain is based on hyperbolic two-temperature heat conduction laws.

The main target of this work is to discuss and study the effects of the fractional-order strain parameter and hyperbolic two-temperature parameter on thermomechanical waves through a thermoelastic body.

\section{The governing equations}

Consider a perfect thermal conducting, isotropic, elastic, and cylindrical body occupies the region $\Lambda=\{(r, \psi, z): 0 \leq r \leq a, 0 \leq \psi \leq 2 \pi, 0 \leq z<\infty\}$. We apply a cylindrical coordinates system $(r, \psi, z ; t)$ in which the $z$-axis was lying along the axis of the cylinder, as in Fig. 1 , and due to symmetry, the medium is one-dimensional. Moreover, all the state-functions will depend on the radial distance $r$ and the time $t$. It is proposed that there are no body forces or heat sources in the medium and initially at rest.

Thus, the governing equations in cylindrical one-dimensional with fractional order strain can be written as $[8,24]$.

The equation of motion:

$(\lambda+2 \mu)\left(1+\tau^{\alpha} D_{t}^{\alpha}\right) \frac{\partial e}{\partial r}-\gamma \frac{\partial T}{\partial r}=\rho \frac{\partial^{2} u}{\partial t^{2}}$

The heat equation:

$\nabla^{2} \varphi=\left(\frac{\partial}{\partial t}+\tau_{o} \frac{\partial^{2}}{\partial t^{2}}\right)\left[\frac{\rho C_{E}}{K} \theta+\frac{T_{o} \gamma}{K}\left(1+\tau^{\alpha} D_{t}^{\alpha}\right) e\right]$

$\frac{\partial^{2}}{\partial t^{2}}(\varphi-\theta)=c^{2} \nabla^{2} \varphi$,

where $c(\mathrm{~m} / \mathrm{s})$ is constant (two-temperature parameter).

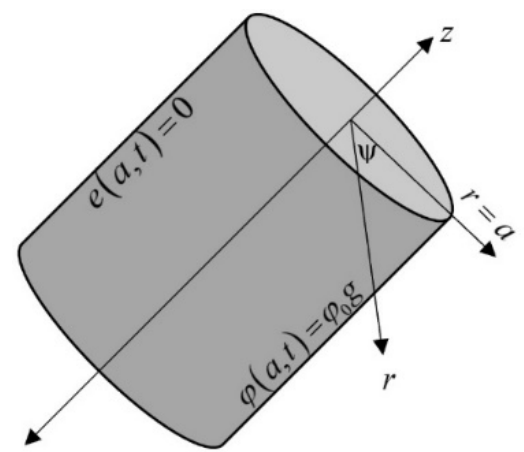

Fig. 1. The isotropic homogeneous thermoelastic solid cylinder

The constitutive relations will take the forms:

$$
\begin{aligned}
& \sigma_{r r}=2 \mu\left(1+\tau^{\alpha} D_{t}^{\alpha}\right) \frac{\partial u}{\partial r}+\lambda\left(1+\tau^{\alpha} D_{t}^{\alpha}\right) e-\gamma \theta \\
& \sigma_{\psi \psi}=2 \mu\left(1+\tau^{\alpha} D_{t}^{\alpha}\right) \frac{u}{r}+\lambda\left(1+\tau^{\alpha} D_{t}^{\alpha}\right) e-\gamma \theta \\
& \sigma_{z z}=\lambda\left(1+\tau^{\alpha} D_{t}^{\alpha}\right) e-\gamma \theta \\
& \sigma_{z r}=\sigma_{\psi r}=\sigma_{z z}=0
\end{aligned}
$$


and $e$ is the volume dilatation and satisfies the relation:

$e=\frac{1}{r} \frac{\partial(r u)}{\partial r}$

where $\nabla^{2}=\frac{\partial^{2}}{\partial r^{2}}+\frac{1}{r} \frac{\partial}{\partial r}$.

In the above equations, we apply the definition of the Riemann-Liouville fractional integral $I^{\alpha} f(t)$ written in a convolution type form [25]:

$I^{\alpha} f(t)=\frac{1}{\Gamma(\alpha)} \int_{0}^{t}(t-v)^{\alpha-1} f(v) d v, \quad t>0, \quad \alpha>0$,

which gives Caputo fractional derivatives in the form:

$D_{t}^{\alpha} f(t)=I^{-\alpha} f(t)=\frac{1}{\Gamma(1-\alpha)} \int_{0}^{t}(t-v)^{-\alpha} f(v) d v, \quad t>0,1>\alpha>0$.

For convenience, we will use the following non-dimensional variables [8, 24]:

$\left\{r^{\prime}, u^{\prime}, a^{\prime}\right\}=c_{o} \eta\{r, u, a\}, \quad\left\{t^{\prime}, \tau^{\prime}, \tau_{o}^{\prime}\right\}=c_{o}^{2} \eta\left\{t, \tau, \tau_{o}\right\}, \quad\left\{\theta^{\prime}, \varphi^{\prime}\right\}=\frac{1}{T_{0}}\{\theta, \varphi\}, \quad \sigma^{\prime}=\frac{\sigma}{\mu^{\prime}}$

where $c_{o}^{2}=\frac{\lambda+2 \mu}{\rho}$ and $\eta=\frac{\rho C_{E}}{K}$.

Eqs. (1)-(6) take the following forms (the primes are suppressed for simplicity):

$\left(1+\tau^{\alpha} D_{t}^{\alpha}\right) \nabla^{2} e-b \nabla^{2} \theta=\frac{\partial^{2} e}{\partial t^{2}}$

$\nabla^{2} \varphi=\left(\frac{\partial}{\partial t}+\tau_{o} \frac{\partial^{2}}{\partial t^{2}}\right)\left[\theta+\varepsilon\left(1+\tau^{\alpha} D_{t}^{\alpha}\right) e\right]$,

$\frac{\partial^{2}}{\partial t^{2}}(\varphi-\theta)=\tilde{c}^{2} \nabla^{2} \varphi$,

$\sigma_{r r}=\beta^{2}\left(1+\tau^{\alpha} D_{t}^{\alpha}\right) e-2\left(1+\tau^{\alpha} D_{t}^{\alpha}\right) \frac{u}{r}-\varepsilon_{1} \theta$,

$\sigma_{\psi \psi}=\beta^{2}\left(1+\tau^{\alpha} D_{t}^{\alpha}\right) e-2\left(1+\tau^{\alpha} D_{t}^{\alpha}\right) \frac{\partial u}{\partial r}-\varepsilon_{1} \theta$,

$\sigma_{z z}=\left(\beta^{2}-2\right)\left(1+\tau^{\alpha} D_{t}^{\alpha}\right) e-\varepsilon_{1} \theta$,

where $\gamma=(3 \lambda+2 \mu) \alpha_{T}, \varepsilon_{1}=\frac{\gamma T_{o}}{\mu}, \varepsilon=\frac{\gamma}{\rho C_{E}}, \beta=\left(\frac{\lambda+2 \mu}{\mu}\right)^{1 / 2}, b=\frac{\varepsilon_{1}}{\beta^{2}}, \tilde{c}^{2}=\frac{c^{2}}{c_{o}^{2}}$.

We apply the Laplace transform defined as:

$\ell\{f(t)\}=\bar{f}(s)=\int_{0}^{\infty} f(t) e^{-s t} d t$

The Laplace transform of the fractional derivative is defined as [25]:

$\ell\left\{D_{t}^{\alpha} f(t)\right\}=s^{\alpha} \bar{f}(s)-D_{t}^{\alpha} I^{1-\alpha} f\left(0^{+}\right), \quad 0<\alpha<1$.

We assume the following initial conditions: 
$D_{t}^{\alpha} I^{1-\alpha}\left\{u\left(r, 0^{+}\right), e\left(r, 0^{+}\right)\right\}=0, \quad 0<\alpha<1$,

$\theta(r, 0)=\varphi(r, 0)=\left.\frac{\partial \theta(r, t)}{\partial t}\right|_{t=0}=\left.\frac{\partial \varphi(r, t)}{\partial t}\right|_{t=0}$.

Thus, the Eqs. (12)-(17) have the forms:

$\left(1+\tau^{\alpha} s^{\alpha}\right) \nabla^{2} \bar{e}-b \nabla^{2} \bar{\theta}=s^{2} \bar{e}$

$\nabla^{2} \bar{\varphi}=\left(s+\tau_{o} s^{2}\right)\left[\bar{\theta}+\varepsilon\left(1+\tau^{\alpha} s^{\alpha}\right) \bar{e}\right]$,

$\bar{\varphi}-\bar{\theta}=\delta^{2} \nabla^{2} \bar{\varphi}$,

$\bar{\sigma}_{r r}=\beta^{2}\left(1+\tau^{\alpha} s^{\alpha}\right) \bar{e}-2\left(1+\tau^{\alpha} s^{\alpha}\right) \frac{\bar{u}}{r}-\varepsilon_{1} \bar{\theta}$,

$\bar{\sigma}_{\psi \psi}=\beta^{2}\left(1+\tau^{\alpha} s^{\alpha}\right) \bar{e}-2\left(1+\tau^{\alpha} s^{\alpha}\right) \frac{\partial \bar{u}}{\partial r}-\varepsilon_{1} \bar{\theta}$,

$\bar{\sigma}_{z z}=\left(\beta^{2}-2\right)\left(1+\tau^{\alpha} S^{\alpha}\right) \bar{e}-\varepsilon_{1} \bar{\theta}$,

$\bar{e}=\frac{1}{r} \frac{\partial(r \bar{u})}{\partial r}=\frac{\bar{u}}{r}+\frac{\partial \bar{u}}{\partial r^{\prime}}$

where $\delta^{2}=\tilde{c}^{2} / s^{2}$.

We can re-write Eqs. (22)-(24) in the forms:

$\nabla^{2} \bar{e}-\alpha_{1} \nabla^{2} \bar{\theta}=\alpha_{2} \bar{e}$,

$\nabla^{2} \bar{\varphi}=\alpha_{3} \bar{\theta}+\alpha_{4} \bar{e}$,

$\bar{\theta}=\bar{\varphi}-\delta^{2} \nabla^{2} \bar{\varphi}$,

where $\alpha_{1}=\frac{b}{\left(1+\tau^{\alpha} s^{\alpha}\right)}, \alpha_{2}=\frac{s^{2}}{\left(1+\tau^{\alpha} s^{\alpha}\right)}, \alpha_{3}=\left(s+\tau_{o} s^{2}\right), \alpha_{4}=\varepsilon\left(s+\tau_{o} s^{2}\right)\left(1+\tau^{\alpha} s^{\alpha}\right)$.

By inserting Eq. (31) into the Eq. (29), we obtain:

$\left(\nabla^{2}-\alpha_{2}\right) \bar{e}=\alpha_{1}\left(\nabla^{2}-\delta^{2} \nabla^{4}\right) \bar{\varphi}$.

Substitute from Eq. (31) into the Eq. (30), we get:

$\left(\nabla^{2}-\alpha_{5}\right) \bar{\varphi}=\alpha_{6} \bar{e}$,

where $\alpha_{5}=\frac{\alpha_{3}}{\left(1+\alpha_{3} \delta^{2}\right)}$ and $\alpha_{6}=\frac{\alpha_{4}}{\left(1+\alpha_{3} \delta^{2}\right)}$.

Substitute from Eq. (33) into the Eq. (31), we get:

$\bar{\theta}=\alpha_{7} \bar{\varphi}-\alpha_{8} \bar{e}$,

where $\alpha_{7}=\left(1-\delta^{2} \alpha_{5}\right)$ and $\alpha_{8}=\delta^{2} \alpha_{6}$.

By eliminating $\bar{e}$ between Eq. (32) and (33), we obtain:

$\left(\nabla^{4}-L \nabla^{2}+M\right) \bar{\varphi}=0$.

By eliminating $\bar{\varphi}$ between Eqs. (32) and (33), we get:

$\left(\nabla^{4}-L \nabla^{2}+M\right) \bar{e}=0$,

where $L=\frac{\left(\alpha_{2}+\alpha_{5}+\alpha_{1} \alpha_{6}\right)}{\left(1+\delta^{2} \alpha_{1} \alpha_{6}\right)}$ and $M=\frac{\alpha_{2} \alpha_{5}}{\left(1+\delta^{2} \alpha_{1} \alpha_{6}\right)}$.

The bounded solutions at $r=0$ of the Eqs. (35) and (36) take the forms: 
$\varphi(r, s)=\alpha_{6} \sum_{i=1}^{2} A_{i} I_{0}\left(k_{i} r\right)$

$\bar{e}(r, s)=\sum_{i=1}^{2}\left(k_{i}^{2}-\alpha_{5}\right) A_{i} I_{0}\left(k_{i} r\right)$

where $I_{0}(*)$ is the modified Bessel function of the first kind of order zero, and $\pm k_{1}, \pm k_{2}$ are the roots of the characte, ristic equation:

$k^{4}-L k^{2}+M=0$.

For the thermal boundary condition, we consider the edge of the cylinder $r=a$ to be thermally loaded as follows:

$\varphi(a, t)=\varphi_{o} g(t)$,

where $g(t)$ is the function of the thermal loading and $\varphi_{o}$ is constant (the thermal loading strength). For the mechanical boundary conditions, we consider the edge of the cylinder $r=a$ is connected to a rigid foundation which can prevent any displacement which leads to zero volumetric deformation as follows:

$e(a, t)=0$.

Applying Laplace transform on the Eqs. (40) and (41), we obtain:

$\bar{\varphi}(a, s)=\varphi_{o} \bar{g}(s)$.

Applying the boundary conditions in the Eqs. (37) and (38), we get the following system:

$\sum_{i=1}^{2} A_{i} I_{0}\left(k_{i} a\right)=\frac{\varphi_{o} \bar{g}(s)}{\alpha_{6}}$,
$\sum_{i=1}^{2}\left(k_{i}^{2}-\alpha_{5}\right) A_{i} I_{0}\left(k_{i} a\right)=0$.

By solving the system in Eq. (43) and Eq. (44), we get:

$A_{1}=\frac{\varphi_{0} G(s)\left(k_{2}^{2}-\alpha_{5}\right)}{\alpha_{6}\left(k_{2}^{2}-k_{1}^{2}\right) I_{0}\left(k_{1} a\right)}, \quad A_{2}=-\frac{\varphi_{0} G(s)\left(k_{1}^{2}-\alpha_{5}\right)}{\alpha_{6}\left(k_{2}^{2}-k_{1}^{2}\right) I_{0}\left(k_{2} a\right)}$.

Hence, we have:

$\varphi(r, s)=\frac{\varphi_{0} G(s)}{\left(k_{2}^{2}-k_{1}^{2}\right)}\left[\frac{\left(k_{2}^{2}-\alpha_{5}\right)}{I_{0}\left(k_{1} a\right)} I_{0}\left(k_{1} r\right)-\frac{\left(k_{1}^{2}-\alpha_{5}\right)}{I_{0}\left(k_{2} a\right)} I_{0}\left(k_{2} r\right)\right]$,

$\bar{e}(r, s)=\frac{\varphi_{0} G(s)\left(k_{2}^{2}-\alpha_{5}\right)\left(k_{1}^{2}-\alpha_{5}\right)}{\alpha_{6}\left(k_{2}^{2}-k_{1}^{2}\right)}\left[\frac{I_{0}\left(k_{1} r\right)}{I_{0}\left(k_{1} a\right)}-\frac{I_{0}\left(k_{2} r\right)}{I_{0}\left(k_{2} a\right)}\right]$.

To get the displacement function, we use the Eqs. (28) and (46) as follows:

$\bar{u}(r, s)=\frac{1}{r} \int r \bar{e}(r, s) d r$, 
which gives:

$$
\begin{aligned}
& \bar{u}(r, s) \\
& \quad=\frac{\varphi_{0} G(s)\left(k_{2}^{2}-\alpha_{5}\right)\left(k_{1}^{2}-\alpha_{5}\right)}{\alpha_{6}\left(k_{2}^{2}-k_{1}^{2}\right)}\left[\frac{1}{I_{0}\left(k_{1} a\right)} \frac{1}{r} \int r I_{0}\left(k_{1} r\right) d r-\frac{1}{I_{0}\left(k_{2} a\right)} \frac{1}{r} \int r I_{0}\left(k_{2} r\right) d r\right] .
\end{aligned}
$$

We use the well-known relation of Bessel integration as follows:

$$
I_{1}(z)=\frac{1}{z} \int z I_{0}(z) d z
$$

where $I_{1}(*)$ is the modified Bessel function of the first kind of first order.

Hence, we obtain:

$$
\bar{u}(r, s)=\frac{\varphi_{0} G(s)\left(k_{2}^{2}-\alpha_{5}\right)\left(k_{1}^{2}-\alpha_{5}\right)}{\alpha_{6}\left(k_{2}^{2}-k_{1}^{2}\right)}\left[\frac{1}{I_{0}\left(k_{1} a\right) k_{1}} I_{1}\left(k_{1} r\right)-\frac{1}{I_{0}\left(k_{2} a\right) k_{2}} I_{1}\left(k_{2} r\right)\right] .
$$

To obtain the dynamical temperature, we substitute from equations (45) and (46) in the Eqs. (34) to be in the form:

$$
\begin{gathered}
\bar{\theta}(r, s)=\frac{\varphi_{0} G(s)}{\alpha_{6}\left(k_{2}^{2}-k_{1}^{2}\right)}\left[\frac{\left(k_{2}^{2}-\alpha_{5}\right)\left[\alpha_{7} \alpha_{6}-\alpha_{8}\left(k_{1}^{2}-\alpha_{5}\right)\right]}{I_{0}\left(k_{1} a\right)} I_{0}\left(k_{1} r\right)\right. \\
\left.-\frac{\left(k_{1}^{2}-\alpha_{5}\right)\left[\alpha_{7} \alpha_{6}-\alpha_{8}\left(k_{2}^{2}-\alpha_{5}\right)\right]}{I_{0}\left(k_{2} a\right)} I_{0}\left(k_{2} r\right)\right] .
\end{gathered}
$$

To get the stress distribution and to avoid the singularity when $r=0$, we take the average of the three principal stresses components as follows:

$\bar{\sigma}=\frac{\bar{\sigma}_{r r}+\bar{\sigma}_{\psi \psi}+\bar{\sigma}_{z Z}}{3}=\left(\beta^{2}-\frac{4}{3}\right)\left(1+\tau^{\alpha} s^{\alpha}\right) \bar{e}-\varepsilon_{1} \bar{\theta}$.

We re-write the Eq. (52) to be in the form:

$\bar{\sigma}=\alpha_{7} \bar{e}-\varepsilon_{1} \bar{\theta}$

where $\alpha_{9}=\left(\beta^{2}-4 / 3\right)\left(1+\tau^{\alpha} s^{\alpha}\right)$.

Substitute from Eqs. (46) and (51) in the Eq. (53), hence, we get:

$$
\begin{gathered}
\bar{\sigma}(r, s)=\frac{\varphi_{0} G(s)}{\alpha_{6}\left(k_{2}^{2}-k_{1}^{2}\right)}\left[\frac{\left(k_{2}^{2}-\alpha_{5}\right)\left(\alpha_{7}\left(k_{1}^{2}-\alpha_{5}\right)-\varepsilon_{1}\left[\alpha_{7} \alpha_{6}-\alpha_{8}\left(k_{1}^{2}-\alpha_{5}\right)\right]\right)}{I_{0}\left(k_{1} a\right)} I_{0}\left(k_{1} r\right)\right. \\
\left.-\frac{\left(k_{1}^{2}-\alpha_{5}\right)\left(\alpha_{7}\left(k_{2}^{2}-\alpha_{5}\right)-\varepsilon_{1}\left[\alpha_{7} \alpha_{6}-\alpha_{8}\left(k_{2}^{2}-\alpha_{5}\right)\right]\right)}{I_{0}\left(k_{2} a\right)} I_{0}\left(k_{2} r\right)\right] .
\end{gathered}
$$

The conducting and dynamical temperature increments, pressure, displacement, and average stress can both be measured using Riemann-sum approximation techniques over time. This method is used to invert some function's Laplace transformation [26]:

$f(t)=\frac{e^{\kappa t}}{t}\left[\frac{1}{2} \bar{f}(\kappa)+\operatorname{Re} \sum_{n=1}^{N}(-1)^{n} \bar{f}\left(\kappa+\frac{i n \pi}{t}\right)\right]$

where " $i$ " is an imaginary number unit, and "Re" is the real part. For convergence with a faster 
process, many numerical experiments have shown that the value $\kappa$ satisfies the relation $\kappa t \approx 4.7[26]$.

\section{Numerical results and discussion}

Copper is known as the thermoelastic material for the numerical results and for the various physical constants we take the following values: $K=386 \mathrm{~kg} \mathrm{~m} \mathrm{k}^{-1} \mathrm{~s}^{-3}, \alpha_{T}=1.78 \times 10^{-5} \mathrm{k}^{-1}$, $\rho=8954 \mathrm{~kg} \mathrm{~m}{ }^{-3}, T_{o}=293 \mathrm{k}, C_{E}=383.1 \mathrm{~m}^{2} \mathrm{k}^{-1} \mathrm{~s}^{-2}, \mu=3.8610^{10} \mathrm{~kg} \mathrm{~m}^{-1} \mathrm{~s}^{-2}$, $\lambda=7.76 \times 10^{10} \mathrm{~kg} \mathrm{~m}^{-1} \mathrm{~s}^{-2}$.

Thus, we get the non-dimensional values of the problem as $[8,24]: b=0.01047$, $\varepsilon_{1}=0.0419, \varepsilon=1.6086, \beta^{2}=4, \varphi_{0}=1.0, \tau_{o}=0.02, \tau=0.01, \tilde{c}=0.1$ (assumed).

The numerical results of the conductive and dynamical temperature increments, the strain, the displacement, and the average stress distributions have been figured for a wide range of the dimensionless radial distance $r(0 \leq r \leq 2.0)$ at the instant value of dimensionless time $t=1.0$.

In Figs. 2-6, the calculations have been carried out for various values of the fractional-order parameter $\alpha=(0.0,0.5,0.9)$ in the context of the hyperbolic two-temperature thermoelasticity model to stand on the effect of this parameter on all the studied functions.

It is noted from Figs. 2 and 3 that the fractional-order parameter has very limited effects on the conductive and dynamical temperature increment. It is expectable results where the fractional-order definition has been applied to the strain function.

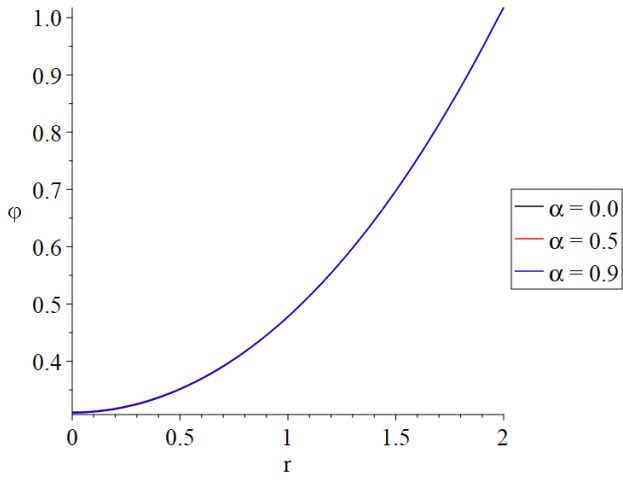

Fig. 2. The conductive temperature distribution with the variance value of the fractional-order parameter

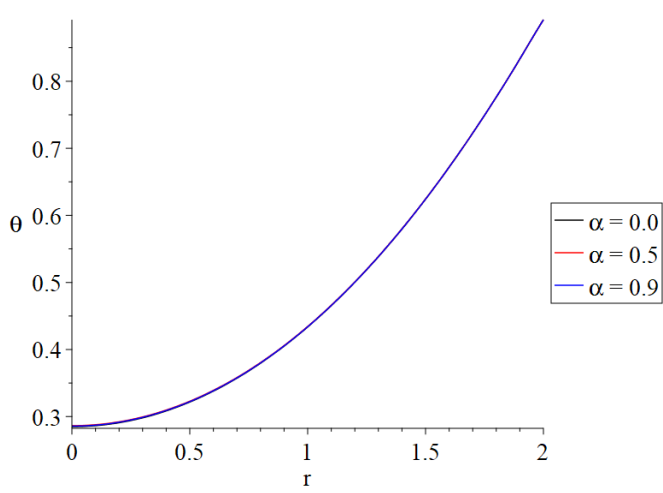

Fig. 3. The dynamical temperature distribution with variance value of fractional order parameter

Fig. 4 shows that the fractional-order parameter has a significant effect on the strain distributions. The absolute values of the peak points of the strain distribution take the following order:

$|e(\alpha=0.0)|>|e(\alpha=0.9)|>|e(\alpha=0.5)|$.

Fig. 5 shows that the fractional-order parameter has a significant effect on the displacement distributions, and this effect is visible near the impact of the thermal shock. The absolute values of the displacement at the edge of the cylinder $r=a$ take the following order:

$|u(\alpha=0.0)|>|u(\alpha=0.9)|>|u(\alpha=0.5)|$.

Fig. 6 shows that the fractional-order parameter has a significant effect on the average stress distributions. The absolute values of the average stress at the center of the cylinder $r=0$ take the following order:

$|\sigma(\alpha=0.5)|>|\sigma(\alpha=0.9)|>|\sigma(\alpha=0.0)|$. 


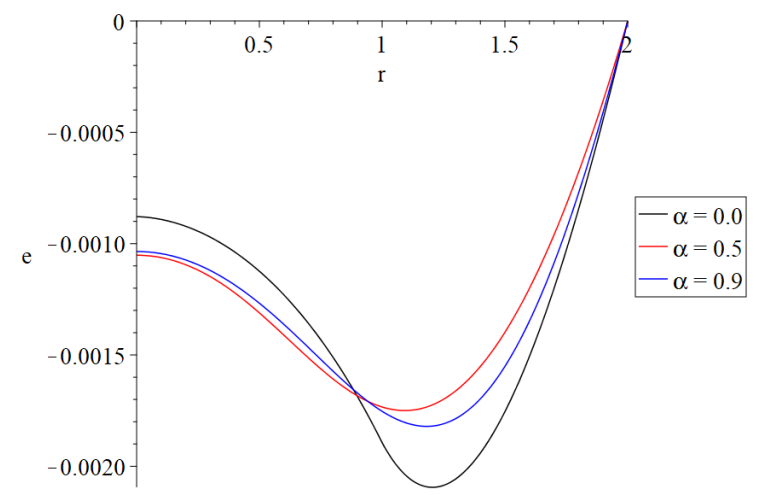

Fig. 4. The strain distribution with variance value of fractional order parameter

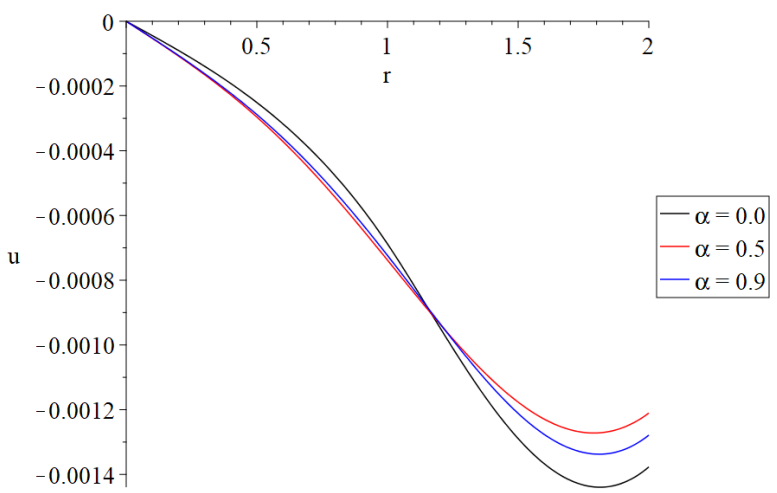

Fig. 5. The displacement distribution with variance value of fractional order parameter

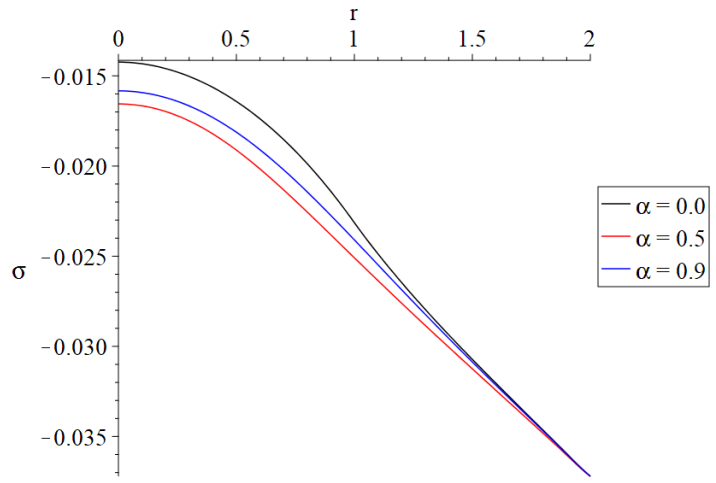

Fig. 6. The average stresses distribution with variance value of fractional order parameter

In Figs. 7-11, the calculations have been carried out for various models of thermoelasticity (one-temperature, two-temperature, and hyperbolic two-temperature) without fractional-order strain $\alpha=0.5$ to stand on the two-temperature parameter effects on all the studied functions.

In Fig. 7, it is noticed that the behavior of the conductive temperature increment distributions in the context of the one-temperature and hyperbolic two-temperature models is closed but with different values. In contrast, the behavior of the classical two-temperature model is different. The curves of the one-temperature and hyperbolic two-temperature models fail down close to zero, more than the curve of the two-temperature model. The one-temperature and hyperbolic two-temperature models offer thermal waves with finite speed of propagation while the model of classical two-temperature parameter offers thermal waves with an infinite speed of propagation. 
Fig. 8 shows that the dynamical temperature increment distributions in the context of the one-temperature and hyperbolic two-temperature models have the same behavior but with different values, while the behavior of the classical two-temperature model is different. The values of the dynamical temperature increment based on the one-temperature model in Fig. 8 coincide with the value of the conductive temperature increment in Fig. 7 based on the same model.

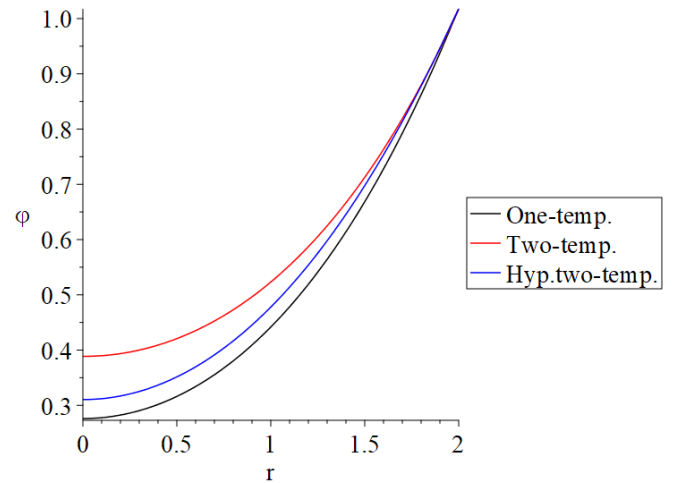

Fig. 7. The conductive temperature increment distribution with variance models and $\alpha=0.5$

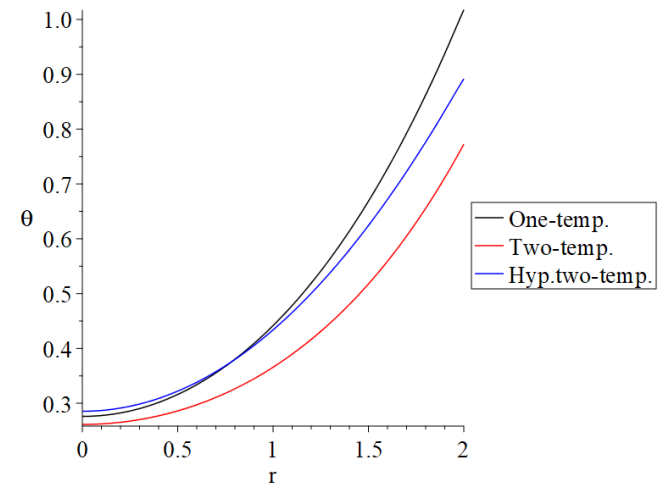

Fig. 8. The dynamical temperature increment distribution with variance models $\alpha=0.5$

Fig. 9 shows that the strain distributions in the context of the one-temperature and hyperbolic two-temperature models have the same behavior but with different values, while its behavior based on the classical two-temperature model is different. The absolute values of the peak points of the strain take the following order:

$\mid e_{\max }($ one - temp $)|>| e_{\max }($ hyp two - temp $)|>| e_{\max }($ two - temp $) \mid$.

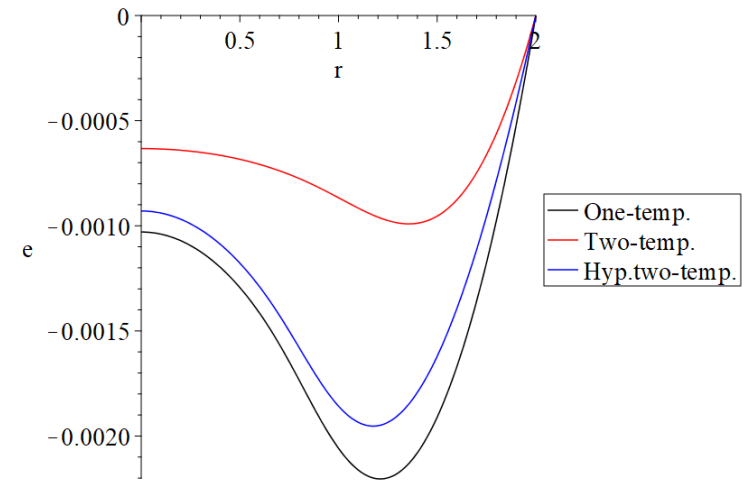

Fig. 9. The strain increment distribution with variance models $\alpha=0.5$

Fig. 10 shows that the displacement distributions in the context of the one-temperature and hyperbolic two-temperature models have closed behavior, while its behavior based on the classical two-temperature model is different. The values of the peak points of the displacement take the following order:

$\mid u_{\max }($ one - temp $)|>| u_{\max }($ hyp two - temp $)|>| u_{\max }($ two - temp) $\mid$.

Fig. 11 shows that the average stress distributions in the context of the one-temperature and hyperbolic two-temperature models have a closed behavior, while its behavior based on the classical two-temperature model is different. The absolute values of the stress in the edge of the 
cylinder $r=a$ take the following order:

$\mid \sigma($ one - temp $)|>| \sigma($ hyp two - temp $)|>| \sigma($ two - temp $) \mid$.

We can see that for the special cases of zero fractional order of strain, one-temperature model, and classical two-temperature model, the results agree with the results in the publications $[8,27,28]$.

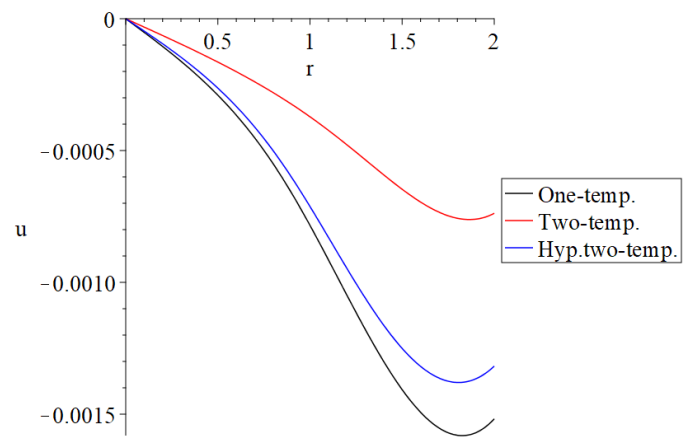

Fig. 10. The displacement distribution with variance models $\alpha=0.5$

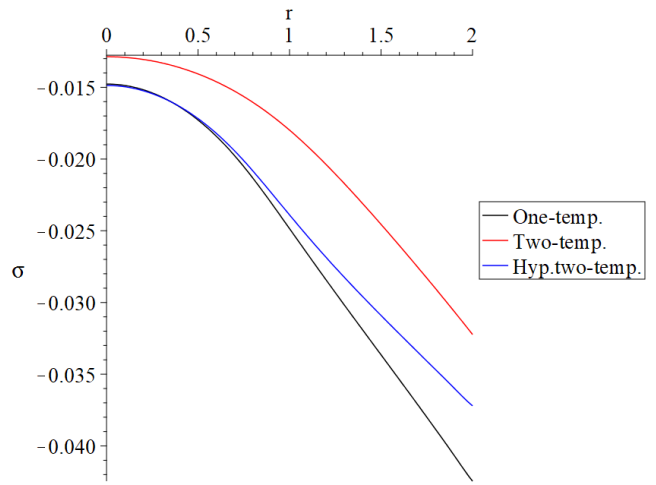

Fig. 11. The average stresses distribution with variance models $\alpha=0.5$

\section{Conclusions}

The issue of a thermoelastic homogeneous isotropic infinite cylindrical medium when the bounding surface is thermally shocked and no volume deformation is addressed in this article. One-temperature, classical two-temperature, and hyperbolic two-temperature models have also been used to solve the problem.

The fractional-order parameter has negligible impacts on conductive and dynamical temperature increments, but has a substantial influence on the strain, displacement, and average stresses, according to the numerical data.

The one-temperature model, like the hyperbolic two-temperature model, produces thermal waves with finite propagation speeds, while the classical two-temperature model generates thermal waves with an infinite propagation speed.

As a result, the hyperbolic two-temperature thermoelasticity model is a good fit for describing the thermodynamic behavior of thermoelastic materials. 


\section{References}

[1] P. J. Chen and M. E. Gurtin, "On a theory of heat conduction involving two temperatures," Zeitschrift für angewandte Mathematik und Physik ZAMP, Vol. 19, No. 4, pp. 614-627, Jul. 1968, https://doi.org/10.1007/bf01594969

[2] W. E. Warren and P. J. Chen, "Wave propagation in the two temperature theory of thermoelasticity," Acta Mechanica, Vol. 16, No. 1-2, pp. 21-33, Mar. 1973, https://doi.org/10.1007/bf01177123

[3] H. M. Youssef, "Theory of two-temperature-generalized thermoelasticity," IMA Journal of Applied Mathematics, Vol. 71, No. 3, pp. 383-390, Jun. 2006, https://doi.org/10.1093/imamat/hxh101

[4] H. M. Youssef and K. A. Elsibai, "On the theory of two-temperature thermoelasticity without energy dissipation of Green-Naghdi model," Applicable Analysis, Vol. 94, No. 10, pp. 1997-2010, Oct. 2015, https://doi.org/10.1080/00036811.2014.961920

[5] I. A. Abbas and H. M. Youssef, "Two-temperature generalized thermoelasticity under ramp-type heating by finite element method," Meccanica, Vol. 48, No. 2, pp. 331-339, Mar. 2013, https://doi.org/10.1007/s11012-012-9604-8

[6] H. Youssef, "A two-temperature generalized thermoelastic medium subjected to a moving heat source and ramp-type heating: a state-space approach," Journal of Mechanics of Materials and Structures, Vol. 4, No. 9, pp. 1637-1649, Jan. 2010, https://doi.org/10.2140/jomms.2009.4.1637

[7] H. M. Youssef, "Two-dimensional problem of a two-temperature generalized thermoelastic half-space subjected to ramp-type heating," Computational Mathematics and Modeling, Vol. 19, No. 2, pp. 201-216, Apr. 2008, https://doi.org/10.1007/s10598-008-0014-7

[8] H. M. Youssef, "Two-temperature generalized thermoelastic infinite medium with cylindrical cavity subjected to moving heat source," Archive of Applied Mechanics, Vol. 80, No. 11, pp. 1213-1224, Nov. 2010, https://doi.org/10.1007/s00419-009-0359-1

[9] H. M. Youssef and A. A. El-Bary, "Theory of hyperbolic two-temperature generalized thermoelasticity," Materials Physics and Mechanics, Vol. 40, No. 2, pp. 158-171, Jan. 2018, https://doi.org/10.18720/mpm.4022018_4

[10] R. L. Magin and T. J. Royston, "Fractional-order elastic models of cartilage: A multi-scale approach," Communications in Nonlinear Science and Numerical Simulation, Vol. 15, No. 3, pp. 657-664, Mar. 2010, https://doi.org/10.1016/j.cnsns.2009.05.008

[11] C. Li, H. Guo, X. Tian, and T. He, "Generalized thermoviscoelastic analysis with fractional order strain in a thick viscoelastic plate of infinite extent," Journal of Thermal Stresses, Vol. 42, No. 8, pp. 1051-1070, Aug. 2019, https://doi.org/10.1080/01495739.2019.1587331

[12] C. Li, H. Guo, X. Tian, and T. He, "Generalized thermoelastic diffusion problems with fractional order strain," European Journal of Mechanics - A/Solids, Vol. 78, p. 103827, Nov. 2019, https://doi.org/10.1016/j.euromechsol.2019.103827

[13] C. Li, H. Guo, X. Tian, and T. He, "Generalized piezoelectric thermoelasticity problems with strain rate and transient thermo-electromechanical responses analysis," ZAMM - Journal of Applied Mathematics and Mechanics / Zeitschrift für Angewandte Mathematik und Mechanik, Vol. 100, No. 5, p. e201900067, May 2020, https://doi.org/10.1002/zamm.201900067

[14] E. M. Hussein, "Fractional order thermoelastic problem for an infinitely long solid circular cylinder," Journal of Thermal Stresses, Vol. 38, No. 2, pp. 133-145, Feb. 2015, https://doi.org/10.1080/01495739.2014.936253

[15] S. Sheoran and P. Kundu, "Fractional order generalized thermoelasticity theories: A review," International Journal of Advances in Applied Mathematics and Mechanics, Vol. 3, No. 4, pp. 76-81, 2016.

[16] M. A. Ezzat, A. S. El-Karamany, and A. A. El-Bary, "Two-temperature theory in Green-Naghdi thermoelasticity with fractional phase-lag heat transfer," Microsystem Technologies, Vol. 24, No. 2, pp. 951-961, Feb. 2018, https://doi.org/10.1007/s00542-017-3425-6

[17] M. A. Ezzat and A. A. El-Bary, "Application of fractional order theory of magneto-thermoelasticity to an infinite perfect conducting body with a cylindrical cavity," Microsystem Technologies, Vol. 23, No. 7, pp. 2447-2458, Jul. 2017, https://doi.org/10.1007/s00542-016-2976-2

[18] N. Sarkar, D. Ghosh, and A. Lahiri, "A two-dimensional magneto-thermoelastic problem based on a new two-temperature generalized thermoelasticity model with memory-dependent derivative," Mechanics of Advanced Materials and Structures, Vol. 26, No. 11, pp. 957-966, Jun. 2019, https://doi.org/10.1080/15376494.2018.1432784 
[19] S. Choudhary, S. Kumar, and J. S. Sikka, "Thermo-mechanical interactions in a fractional order generalized thermoelastic solid with diffusion," Microsystem Technologies, Vol. 23, No. 12, pp. 5435-5446, Dec. 2017, https://doi.org/10.1007/s00542-017-3340-x

[20] M. A. Ezzat, A. S. El-Karamany, and A. A. El-Bary, "Application of fractional order theory of thermoelasticity to 3D time-dependent thermal shock problem for a half-space," Mechanics of Advanced Materials and Structures, Vol. 24, No. 1, pp. 27-35, Jan. 2017, https://doi.org/10.1080/15376494.2015.1091532

[21] M. H. Hendy, M. M. Amin, and M. A. Ezzat, "Application of fractional order theory to a functionally graded perfect conducting thermoelastic half space with variable Lamé's Modulii," Microsystem Technologies, Vol. 23, No. 10, pp. 4891-4902, Oct. 2017, https://doi.org/10.1007/s00542-017-34096

[22] M. A. Ezzat and A. A. El-Bary, "Fractional order theory to an infinite thermo-viscoelastic body with a cylindrical cavity in the presence of an axial uniform magnetic field," Journal of Electromagnetic Waves and Applications, Vol. 31, No. 5, pp. 495-513, Mar. 2017, https://doi.org/10.1080/09205071.2017.1285728

[23] H. M. Youssef, "Theory of generalized thermoelasticity with fractional order strain," Journal of Vibration and Control, Vol. 22, No. 18, pp. 3840-3857, Oct. 2016, https://doi.org/10.1177/1077546314566837

[24] H. M. Youssef, "Generalized thermoelastic infinite medium with cylindrical cavity subjected to moving heat source," Mechanics Research Communications, Vol. 36, No. 4, pp. 487-496, Jun. 2009, https://doi.org/10.1016/j.mechrescom.2008.12.004

[25] Y. Povstenko, Fractional Thermoelasticity. Springer, 2015.

[26] D. Y. Tzou, "A unified field approach for heat conduction from macro - to micro-scales," Journal of Heat Transfer, Vol. 117, No. 1, pp. 8-16, Feb. 1995, https://doi.org/10.1115/1.2822329

[27] Hm Youssef, "Two-temperature generalized thermoelastic infinite medium with cylindrical cavity subjected to different types of thermal loading," WSEAS Transactions on Heat and Mass Transfer, Vol. 1, No. 10, p. 769, Jan. 2006.

[28] H. M. Youssef, "Two-temperature generalized thermoelastic infinite medium with cylindrical cavity subjected to non-gaussian laser beam," Two-Temperature Generalized Thermoelastic Infinite Medium with Cylindrical Cavity Subjected to Non-Gaussian Laser Beam, Vol. 21, No. 2, pp. 222-229, 2016, https://doi.org/10.20855/ijav.2016.21.2415

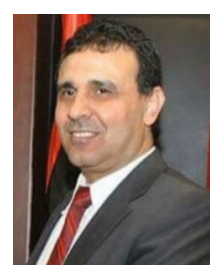

Prof. Alaa AbdelBary research work is centered on developing and integrating innovative statistical and mathematical approaches to advanced physical problems, some of his methodological research focuses on using machine learning for decision support system, Prof. AbdelBary works as a vice president at Arab Academy for Science and Technology (www.aast.edu). Currently, he leads with AASTMT team the sector of higher studies and Scientific Research, AASTMT where a lot of international and national projects and grants in the field of science and technology were obtained. He is a current member in Naqaa Nanotechnology Network and International Leadership Association, and in the editorial boards of several journals including Associate Editor for the J. Applied Science Research, Australian J. Basic and Applied Sciences, International Journal of Basic and Applied Sciences, Research Journal of Applied Sciences, Engineering and Technology, International Journal of Renewable Energy Technology Research, Journal of thermoelasticity, Caspian Journal of Applied Sciences Research and Research Journal of Mathematics and Statistics. Besides, being a reviewer in the following journals: Abstract and Applied Analysis, Applied Mathematics and Computation, Journal of Applied Polymer Sciences, Advances Modeling A, J. Applied Science Research, Australian j. Basic and Applied Sciences and Letters of Applied Mathematics. He published a number of books in the field of mathematics and statistics such as Pure Mathematics with applications (Alexandria, 2002), Financial Mathematics (Alexandria, 2003) and Probability and Statistics (Alexandria, 2006). 